\title{
Spectral classification and distance determination of stars in nine southern Galactic H II regions ${ }^{\star}$ (Research Note)
}

\author{
M. C. Pinheiro, M. V. F. Copetti, and V. A. Oliveira
}

\begin{abstract}
Laboratório de Análise Numérica e Astrofísica, Departamento de Matemática e Programa de Pós-Graduação em Física, Universidade Federal de Santa Maria, 97119-900 Santa Maria, RS, Brazil

e-mail: [pinheiro.marcio;mvfcopetti;prof.vinic]@gmail.com
\end{abstract}

Received 20 July 2009 / Accepted 15 March 2010

\section{ABSTRACT}

\begin{abstract}
Aims. We attempt to identify the ionising stars and to determine the photometric distances of nine southern Galactic $\mathrm{H}$ II regions. Methods. We carried out optical spectroscopy and $U B V$ photometry of the stellar content of these objects. The distance of individual stars were obtained by spectroscopic parallax. To avoid using a fixed value for the total-to-selective extinction ratio $R_{V}$, the reddening $A_{V}$ was determined directly by the colour-difference approach by comparing our $V$ apparent magnitudes and the $J H K$ magnitudes from the 2MASS survey with the intrinsic colour indices.

Results. As types $\mathrm{O}$ or $\mathrm{B}$, we classified 24 of the 31 stars for which optical spectra were obtained. In particular, we identified two new $\mathrm{O}$ stars, one in RCW 98 and the other in RCW 99. The values of reddening obtained correspond to a mean $\left\langle R_{V}\right\rangle=3.44$, which is about $10 \%$ higher than the value found for field stars. For three of the H II regions studied (Bran 186, NGC 2626, and RCW 32), the distance estimates (with errors from $25 \%$ to $50 \%$ ) were based on the data obtained for only one star. For the other six objects (NGC 3503 , NGC 6334, RCW 55, RCW 87, RCW 98, and RCW 99), we obtained more precise photometric distances (with a mean error of $\approx 18 \%$ ) calculated to be the median of the parallax distances obtained for two to six different stars in each nebulae. The parallax distances of individual stars belonging to a given nebula were similar to each other, with internal errors smaller than 5\%, as a consequence of the method used to derive the reddening correction $A_{V}$. The distance of $1.23 \pm 0.30 \mathrm{kpc}$ obtained for RCW 87 disagrees with the value of $7.6 \mathrm{kpc}$ previously found.

Conclusions. The dispersion in individual distance estimates for stars in a given nebula can be significantly reduced by calculating the reddening $A_{V}$ from a comparison between the $V$ and the 2MASS $J H K$ magnitudes instead of using $A_{V}=R_{V} E(B-V)$ with a fixed value for $R_{V}$. Therefore, more precise distances can be calculated with our proposed method.
\end{abstract}

Key words. H II regions - stars: early-type - stars: distances

\section{Introduction}

Several photometric and spectroscopic optical studies of the stellar content of Galactic H II regions have been published (Russeil et al. 2007, and references therein). The ionising stellar associations obscured by interstellar extinction have been intensively studied by observations in the infrared (Borissova et al. 2006). These works have been instrumental in identifying and characterizing the ionising stars, determinations of distance and age of the clusters, defining the spiral structure of the Milky Way, and, indirectly, to estimate the Galactic gradients of chemical abundances. However, the optical studies of relatively small H II regions, ionised by groups of a few stars, remain far from complete. Even the nature of some of these emission nebulae remain unclear or unconfirmed. Some of these objects have even been reclassified (Frew et al. 2006; Copetti et al. 2007; Stupar et al. 2008), but there are several objects with unverified, uncertain or even with no distance determination or classification. In this paper, we present a study of stars in nine southern Galactic $\mathrm{H}$ II regions with discrepant or unknown distance estimates. We performed $U B V$ photometry and optical spectroscopy of these stars to determine their distances using the spectroscopic parallax method and, consequently, the distances of the host nebulae.

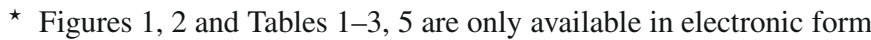
at http://www . aanda.org

\section{Observations}

Direct images in the $U B V$ bands were obtained with the $0.6 \mathrm{~m}$ telescope at the Observatório do Pico dos Dias (OPD), Brazil. To avoid the saturation of the brightest stars, multiple exposures were taken in each filter. Five dome flat-fields for each filter and 15 bias exposures were taken at both the beginning and the end of each night. Images of standard star fields indicated by Landolt (1992) were taken to enable a photometric calibration to be performed. Secondary standard stars catalogued by Galadí-Enríquez et al. (2000) were also used. Table 1 presents the journal of photometric observations. The CCD image fieldof-view was $11^{\prime} \times 11^{\prime}$. The typical seeing ranged from 1'.5 to $22^{\prime \prime} 0$. The reduction followed the standard procedures for stellar CCD photometry in relatively crowed fields and was performed with IRAF. We limited the $U B V$ photometry to stars with $V \leq 18$. Based on the colour-colour and colour-magnitude diagrams, early-type star candidates with $V \leq 14$ were selected for the spectroscopic follow-up observations. For the stars within $13 \leq$ $V \leq 14$, we observed photometric errors of $\sigma_{V}=0.019 \pm 0.011$, $\sigma_{(B-V)}=0.026 \pm 0.015$, and $\sigma_{(U-B)}=0.024 \pm 0.012$.

Long-slit spectrophotometric observations were carried out with the Cassegrain spectrograph attached to the $1.6 \mathrm{~m}$ and the $0.6 \mathrm{~m}$ telescopes at OPD. Table 2 presents the journal of the spectroscopic observations. A Marconi CCD of $2048 \times 2048$ pixels 
was used. The spectra covered the range of 4000 to $4900 \AA$, classically used for spectral classification of OB stars, with signalto-noise ratios ranging from 70 to 400 , with a mean value of about 200. The observation routine followed the usual procedures. Around 15 bias frames and 5 dome flat-fields were taken at the beginning and the end of each night. Spectra of a He-Ar lamp were taken before and after each object exposure to perform the wavelength calibration. The standard reduction procedure was completed with IRAF. We used the splot routine to normalise the continuum of the spectra to unity and to perform spectral line measurements. In each nebula, in general we observed three or four of the hottest and brightest early-type star candidates indicated by the $U B V$ photometry. Since we used a long slit of $1^{\prime \prime} .5 \times 320^{\prime \prime}$, some secondary candidates could be observed simultaneously with the primary targets. Although we could not spectroscopically observe all candidates, the chances of missing the dominant ionising stars is low since these stars should be able to be distinguished from the relatively cool stars, unless they are hidden by local dust lanes.

\section{Methods}

\subsection{Spectral classification}

The spectra of OB stars are easily identified by the presence of He I absorption lines. The presence of He II absorption line is indicative of $\mathrm{O}$ stars. These were the primary criteria used to classify the stars studied here. We measured the equivalent widths of strong absorption lines of the He I, He II, and Balmer series to compare them with the values compiled by Jaschek \& Jaschek (1990) and reduce our search to a few spectral types. For O stars, the He I $\lambda 4471 / \mathrm{He}$ II $\lambda 4541$ ratio and the He I $\lambda 4388 \times$ He II $\lambda 4686$ product were also used in accordance with the prescription by Mathys $(1988,1989)$. Finally, we refined the spectral classification by comparing with the digital spectral atlas of Walborn \& Fitzpatrick (1990), observing the specific characteristics of each spectral subtype individually described by these authors. To classify late B or cooler stars, we relied solely on the average spectral type characteristics presented by Jaschek \& Jaschek (1990). The spectra of the observed OB stars are displayed in Fig. 1.

\subsection{Reddening correction and distance determination}

A major source of error in the photometric distances is the reddening correction, which is often estimated for the $V$ magnitude from the colour excess $E(B-V)$ by using a mean value for the total-to-selective extinction ratio of $R_{V}=A_{V} / E(B-V) \approx 3.1$ (He et al. 1995). However, the extinction of stars inside starforming regions can be anomalous and correspond to different, commonly far higher, values of $R_{V}$ (Mathis 1990; Papaj et al. 1991; Megier et al. 1997; Patriarchi et al. 2001). Thus, the use of a "normal" value of $R_{V}$ valid for field stars can introduce large errors into the estimated distances of H II regions.

In this paper, we chose to evaluate the reddening $A_{V}$ directly by the colour-difference approach comparing our $V$ apparent magnitudes and the $J H K$ magnitudes from the 2MASS survey (Cutri et al. 2003) with the intrinsic colour indices. Using the least squares method, we fitted the relationship

$E(\lambda-V)=A_{V}\left(R_{L}(\lambda)-1\right)$,

where $\lambda$ corresponds to the apparent magnitudes in the $J H K$ filters, the extinction curve $R_{L}(\lambda)=A_{\lambda} / A_{V}$ given by Rieke \& Lebofsky (1985), and the intrinsic colours $(J-V)_{0},(H-V)_{0}$, and $(K-V)_{0}$ from Wegner (1994).
The extinction law is approximately described by a power law with a fixed exponent $\beta$. However, Fitzpatrick \& Massa (2009) argued that there may not be a universal law for the extinction curve, which should depend instead on the sight line. The power law approximation would remain valid, but would different values of $\beta$ for different sight lines (and wavelength ranges). Based on the dispersion of $\approx 20 \%$ in the values of $\beta$ fitted by Fitzpatrick \& Massa (2009), we found that the usual adoption of a extinction curve with a fixed exponent would cause errors in distance of $\approx 15 \%$.

The heliocentric photometric distance $d$ was calculated adopting the calibration of the absolute magnitude $M_{V}$ as a function of the spectral type and luminosity class given by Russeil (2003). The error in distance was estimated to be

$\sigma_{d}=\frac{\ln 10}{5} d \sqrt{\sigma_{V}^{2}+\sigma_{M_{V}}^{2}+\sigma_{A_{V}}^{2}}$

where $\sigma_{V}, \sigma_{M_{V}}$ and $\sigma_{A_{V}}$ are the uncertainties in apparent and absolute magnitudes and in reddening, respectively. The dominant sources of the errors in distance are uncertainties in the absolute magnitude adopted and in reddening, since $0.1 \lessgtr \sigma_{A_{V}} \lesssim 1.0$, and $\sigma_{M_{V}} \approx 0.5$ (Russeil 2003), whereas $\sigma_{V} \lesssim 0.05$.

\section{Results and discussions}

Table 3 presents the results of our spectroscopic and photometric studies of individual stars. Columns 1-3 provide the identifications of the stars studied in this paper, in the 2MASS survey, and in the Simbad database, respectively. Columns 4 and 5 indicate the stellar spectral classifications from this paper and from the literature, respectively. Columns 7 to 12 show the observed $V$ magnitude and $(B-V)$ colour, the colour excess $E(B-V)$, the calculated reddening $A_{V}$, the total-to-selective extinction ratio $R_{V}$, and the corresponding heliocentric photometric distance $d$, respectively.

Table 4 indicates the photometric distance of each H II region studied, which corresponds to the median of the values obtained for $n$ individual stars. For those objects in which more than one star was studied, the error estimates correspond to $\left(\sum \sigma_{i}^{2} / n(n-1)\right)^{1 / 2}$, where $\sigma_{i}$ is the error in the distance for each star. These errors are in the range of $13 \%$ to $50 \%$, and are about $15 \%$ for the objects with three or more stars studied. The standard deviations in the distances measured for different stars in a given nebula are much smaller, varying from $1 \%$ to $13 \%$, but are unrealistic error bounds, serving only to assess the internal consistency of the distance determinations of individual stars, because systematic errors are not taken into account.

To test the completeness of the sample of ionising stars identified in each nebula, we indicate and compare in Table 5, the $\mathrm{H} \beta$ extinction data for both the nebulae and the stars, i.e., $C(\mathrm{H} \beta)$, and for the Lyman continuum photon flux, $N_{\mathrm{c}}$. Columns 3-5 provide the values of $C(\mathrm{H} \beta)$ calculated from the stellar photometry, which are given by $C(\mathrm{H} \beta)=1.5 E(B-V)$, from the nebular $\mathrm{H} \gamma / \mathrm{H} \beta$ line ratio, and from the ratio of the radio continuum flux density to the $\mathrm{H} \beta$ flux, respectively. Columns $6-8$ provide the values of $N_{\mathrm{c}}$ obtained from the summed ionising photon rates of individual stars, from the observed $\mathrm{H} \beta$ line fluxes, and from the radio continuum flux densities at $2.7 \mathrm{GHz}$ (Paladini et al. 2003), respectively. We adopted the values of $N_{\mathrm{c}}$ found by Schaerer \& de Koter (1997) for stars of different spectral types. The integrated $\mathrm{H} \beta$ line fluxes from Copetti (2000) were adopted for all objects apart from Bran 186, for which the extrapolation given by Acker et al. (1991) was used. As an additional compatibility test between the nebular and stellar properties, Table 5 also compares the various spectral types of the identified ionising stars 
Table 4. Heliocentric distances for the studied H II regions.

\begin{tabular}{llll}
\hline \hline H II region & $n$ & \multicolumn{2}{c}{$d(\mathrm{kpc})$} \\
\cline { 3 - 4 } & & This work & Literature \\
\hline Bran 186 & 1 & $3.05 \pm 1.45$ & \\
NGC 2626 & 1 & $1.20 \pm 0.34$ & $0.95[1], 1.25[2]$ \\
RCW 32 & 1 & $0.67 \pm 0.17$ & $0.72[2], 0.70[3], 0.77[4]$ \\
NGC 3503 & 5 & $2.85 \pm 0.37$ & $2.6[1], 3.5[5], 4.2[6]$ \\
RCW 55 & 7 & $4.36 \pm 0.46$ & $4.3[7], 3.08[8]$ \\
RCW 87 & 2 & $1.23 \pm 0.30$ & $7.6[9]$ \\
RCW 98 & 3 & $2.61 \pm 0.46$ & $2.87[2], 3.0[7], 2.8[10]$ \\
RCW 99 & 2 & $3.55 \pm 1.82$ & $3.54[7]$ \\
NGC 6334 & 6 & $1.75 \pm 0.26$ & $2.10[7], 2.3[11], 1.74[12]$ \\
\hline
\end{tabular}

References. [1] Herbst (1975), [2] Crampton \& Fisher (1974), [3] Georgelin et al. (1973), [4] Vogt \& Moffat (1975), [5] Kharchenko et al. (2005), [6] Moffat \& Vogt (1975), [7] Avedisova \& Kondratenko (1984), [8] Brand \& Blitz (1993), [9] Borissova et al. (2006), [10] Yamaguchi et al. (1999), [11] Walborn (1982), [12] Neckel (1978).

and the emission line ratio [O III] $\lambda 4959 / \mathrm{H} \beta$, which roughly assesses the degree of excitation of the nebula. With the exception of one object, RCW 87, the identified ionising stars are capable of maintaining the ionisation of their respective nebulae. The line ratios [O III] $\lambda 4959 / \mathrm{H} \beta$ observed are typical of nebulae excited by early B or late O-type stars. In the following, we comment on some of the objects studied.

NGC 6334. This is a 50' complex of H II regions. We used it to test the distance determination based on $A_{V}$ obtained by comparing the $V$ and $J H K$ magnitudes. We identified the B0.5 V star LS 4087 as the ionising source of the component Gum 64a and reanalysed the data obtained by Walborn (1982) for five other stars in different components of the complex. The mean distance of $1.75 \mathrm{kpc}$ obtained for these six stars exhibited an internal error of only $3.8 \%$. This distance is $24 \%$ lower than the value obtained by Walborn (1982) using a fix value of $R_{V}=3.0$, because of the higher values of $A_{V}$ that we found (corresponding to a mean of $\left.\left\langle R_{V}\right\rangle=3.5\right)$.

NGC 3503. The main sources of ionisation of this H II region are one $\mathrm{B} 0 \mathrm{~V}$ and three $\mathrm{B} 2 \mathrm{~V}$ stars found at the centre of the compact open cluster Pismis 17. The distance estimates for these four stars are remarkably similar, with a mean deviation of less than $2 \%$.

Bran 186. This is a $3^{\prime}$ roughly round low surface brightness emission nebula discovered by Kohoutek (1971) on a $R$ plate of the Palomar Sky Survey, designated as PN K 2-15, and classified as a possible planetary or diffuse nebula. It also appeared as the suspected planetary ESO 260-8 on the ESO/Uppsala survey of the ESO (B) atlas by Holmberg et al. (1978, the original identification was 260-PN?08) and PN G263.2+00.4 on the StrasbourgESO Catalogue of Galactic Planetary Nebulae by Acker et al. (1992). Based on a spectroscopic analysis, Kohoutek \& Pauls (1994) included PN K 2-15 among their list of confirmed planetary nebulae. However, the status of H II region for Bran 186 was strongly indicated by the detection of a site of recent star formation at the eastern border of the optical nebula, 1.8' from the centre, towards the source IRAS 08470-4243, which has IRAS colours typical of ultracompact $\mathrm{H}$ II regions. An embedded star cluster, named [DBS2003] 23, was detected by the 2MASS survey (Dutra et al. 2003) at the position of IRAS 08470-4243.

We found that the B0 V star Bran 186-3 is the ionisation source of Bran 186. A star of this spectral type is compatible with the inferred ionising photon rate and the low degree of excitation of the nebula. In our nebular spectra, we measured [O III] $] 44959 / \mathrm{H} \beta \lesssim 0.17$ and found no helium emission lines, indicating that most of the oxygen is single ionised and the helium is almost neutral.

NGC 2626. This is a bright reflection nebula located at the southwest edge of the large $\left(100^{\prime} \times 100^{\prime}\right) \mathrm{H}$ II region RCW 27 and illuminated by the B1 V star CD-40 4427. For CD-40 4427, we estimated a distance of $1.20 \pm 0.34 \mathrm{kpc}$. For the emissionline star CPD-40 2663 located towards the dark cloud region $4^{\prime}$ from the centre of NGC 2626, a distance of $1.26 \mathrm{kpc}$ was obtained by Gahm \& Malmort (1980). For HD 73882, which is the exciting star of RCW 27, a distance of $1.13 \mathrm{kpc}$ was estimated by Georgelin \& Georgelin (1970a) and $1.04 \mathrm{kpc}$ by Crampton \& Fisher (1974). The similarity between the distance estimates for these three stars indicates that RCW 27, NGC 2626, and the nearby star-formation area are part of the same system.

RCW 55. We classified one star as $\mathrm{O} 8 \mathrm{~V}$ and another six stars as type B. Although the distance estimates for these seven stars are, within the errors, compatible to one another, we may be tempted to consider that there are two superimposed stellar groups, one at $4.65 \pm 0.74 \mathrm{kpc}$ and another at $2.87 \pm 0.59 \mathrm{kpc}$. However, this idea is not supported by the values of $E(B-V)$, which are indicative of similar interstellar extinction.

In Table 5, we see that the total rate of ionising photon from the identified stars is much higher (by two orders of magnitude) than that needed to maintain the ionisation of the nebula. This discrepancy may be caused by a massive leakage of Lyman continuum radiation from the nebula or else by an underestimation of the ionising luminosity required. The $\mathrm{H} \beta$ emission line flux used in this calculation was measured in a circular aperture with $1^{\prime} 40^{\prime \prime}$ of diameter centred on the star RCW 55-7, located in the middle of the bright core of the nebula, while fainter emission extends across a diameter of about $10^{\prime}$. However, a rough extrapolation of the ionising photon flux to the whole nebula based on an image from the AAO/UKST H $\alpha$ survey (Parker et al. 2005) still indicates a value lower (by an order of magnitude) than that from the stars. It is possible than some of the hot stars identified towards RCW 55 are not ionising the nebula, even though they belong to the same stellar association. It is noticeable that the bright nebular core surrounds the B1 V stars RCW 55-3 and RCW 55-7 and not the O8 V star RCW 55-4, the hottest star towards RCW 55. These two B1-type stars are capable of providing the needed ionising radiation.

RCW 87. We classified two 11th magnitude stars as B4 V and B9 III. According to Table 5, these stars are not the main ionising stars of this nebula. Neither of them is in the brightest part of the nebula, where only three or more magnitudes fainter stars are found. The mean distance of these two stars is $d=1.23 \pm$ $0.30 \mathrm{kpc}$. Figure 2 shows the $B-V$ vs. $V$ colour-magnitude diagram for RCW 87. The bulk of stars towards RCW 87 correspond to points in this diagram that are compatible with main sequence stars at the distance and reddening calculated for the two stars that were spectroscopically observed. The stars in the embedded cluster [BDB2003] G320.15+00.79 (Bica et al. 2003), located towards the brightest part of RCW 87, are detached from the rest. However, assuming that these are also main sequence stars, we obtained from the $U-B$ vs. $B-V$ diagram a reddening of $A_{V}=6.8$, leading us to conclude that they are at the same distance as the other stars. Our photometric spectral classification study indicates that there are one B1 and three B3 stars in 
[BDB2003] G320.15+00.79, which could maintain with the ionisation of this nebula.

Borissova et al. (2006) presented a near and mid-infrared study of the star cluster [BDB2003] G320.15+00.79. Based on the spectroscopic parallax of the brightest star of this cluster in the near infrared, which was classified as a K0.5 II type star, these author determined a distance of $7.6 \mathrm{kpc}$ for RCW 87. However, using their distance and reddening of $A_{V}=10.9$ we calculate an ionising photon rate of $\log N_{\mathrm{c}}=51.5 \mathrm{~s}^{-1}$, which is unrealistically high since it would place RCW 87 among the brightest $\mathrm{H}$ II regions of the Galaxy and indicate that this object should be more conspicuous at radio frequencies.

RCW 99. This is a bright $4^{\prime}$ nebula. Avedisova \& Kondratenko (1984) attributed this to a distance of $3.54 \mathrm{kpc}$, a value almost identical to that of $3.55 \mathrm{kpc}$ found in this papers. However, these authors based their result on five stars outside the nebulosity, 28' to $40^{\prime}$ far from its centre. The similarity between the distances of RCW 99 and stars in the field is probably not coincidental but an indication that these stars belong to same large stellar association. No hot star towards the nebulosity had been previously identified. We discovered an O7 III star (RCW 99-1) and a B2 V star (RCW 99-2) in this object.

\section{Summary}

We carried out spectroscopic and photometric observations of the stellar content of 9 nine southern Galactic H II regions to identify ionising stars and to determine the photometric distances of the nebulae. The distance of individual stars were obtained by spectroscopic parallax. To avoid using a fixed value for the total-to-selective extinction ratio $R_{V}$, the reddening $A_{V}$ was determined directly by applying the colour-difference approach comparing our $V$ apparent magnitudes with the $J H K$ magnitudes from the 2MASS survey. Our main findings are:

1. We classified 24 of the 31 stars for which optical spectra were obtained as types $\mathrm{O}$ or $\mathrm{B}$. We identified two new $\mathrm{O}$ stars: RCW 98-2 (O9.5 V) and RCW 99-1 (O7 III).

2. For three of the H II regions studied (Bran 186, NGC 2626, and RCW 32) the distance estimates (with errors from $25 \%$ to $50 \%$ ) were based on the data obtained for only one star. For the other six objects (NGC 3503, NGC 6334, RCW 55, RCW 87, RCW 98, and RCW 99), we obtained more precise photometric distances (with a mean error of $\approx 18 \%$ ) calculated to be the median values of the parallax distances obtained for two to six different stars in each nebulae.

3. The parallax distances of individual stars belonging to a given nebula were found to be similar to each other, having an internal dispersion of less than $5 \%$. This is a consequence of the method used to derive the distance, which is based on an estimation of the reddening correction $A_{V}$ derived by comparing our $V$ magnitude with the $J H K$ magnitudes from 2MASS, and assumes that the total-to-selective extinction ratio of $R_{V}$ is a free parameter.

4. The common procedure of adopting a fixed value for $R_{V}$ to calculate $A_{V}$ from the colour excess $E(B-V)$ would have considerably increased the scatter in the distance estimates and should be employed with restraint in the case of starforming regions.

5. The mean $\left\langle R_{V}\right\rangle=3.46$ was about $10 \%$ higher than the value found for field stars. Stars located more centrally in the nebula tend to exhibit higher values of $R_{V}$.
6. For RCW 87, the distance of $1.23 \pm 0.30 \mathrm{kpc}$ obtained in this paper disagrees with the photometric distance of $7.6 \mathrm{kpc}$ estimated by Borissova et al. (2006) based on a near infrared study of a K0.5 II type star. Additional observations are needed to resolve this discrepancy.

Acknowledgements. This work was supported by the Brazilian agencies CAPES and CNPq. We wish to thank the staff of the Laboratório Nacional de Astrofísica for their assistance during the observations.

\section{References}

Acker, A., Raytchev, B., Stenholm, B., \& Tylenda, R. 1991, A\&AS, 90, 89

Acker, A., Marcout, J., Ochsenbein, F., Stenholm, B., \& Tylenda, R. 1992, Garching: European Southern Observatory

Avedisova, V. S., \& Kondratenko, G. I. 1984, Nauchnye Informatsii, 56, 59

Bica, E., Dutra, C. M., \& Barbuy, B. 2003, A\&A, 397, 177

Borissova J., Ivanov V. D., Minitti D., \& Geisler D. 2006, A\&A, 455, 923

Brand, J., \& Blitz, L. 1993, A\&A, 275, 67

Buscombe, W. 1969, MNRAS, 144, 31

Copetti, M. V. F. 2000, A\&AS, 147, 93

Copetti, M. V. F., Oliveira, V. A., Riffel, R., Castañeda, H. O., \& Sanmartim, D. 2007, A\&A, 472, 847

Corben, P. M., Carter, B. S., Banfield, R. M., \& Harvey, G. M. 1972, MNRAS Afr., 31, 7

Crampton, D. 1971, AJ, 76, 260

Crampton, D., \& Fisher, W. A. 1974, Publ. Dom. Astrophys. Obs., 14, 283

Cutri, R. M., et al. 2003, 2MASS All-Sky Point Source Catalog

Denoyelle, J. 1977, A\&AS, 27, 343

Dutra, C. M., Bica, E., Soares, J., \& Barbuy, B. 2003, A\&A, 400, 533

Fitzpatrick, E. L., \& Massa, D. 2009, ApJ, 699, 1209

Frew, D. J., Parker, Q. A., \& Russeil, D. 2006, MNRAS, 372, 1081

Gahm, G. F., \& Malmort, A. M. 1980, A\&A, 82, 295

Galadí-Enríquez, D., Trullols, E., \& Jordi C. 2000, A\&AS, 146, 169

Georgelin, Y. P., \& Georgelin, Y. M. 1970a, A\&A, 6, 349

Georgelin, Y. P., \& Georgelin, Y. M. 1970b, A\&AS, 3, 1

Georgelin, Y. M., Georgelin, Y. P., \& Roux, S. 1973, A\&A, 25, 337

He, L., Whittet, D. C. B., KilKenny, D., \& Spencer Jones, J. H. 1995, ApJS, 101, 335

Herbst, W. 1975, AJ, 80, 212

Holmberg, E. B., Lauberts, A., Schuster, H.-E., \& West, R. M. 1978, A\&AS, 31, 15

Houk, N. 1978, Michigan Catalog of Two-dimensional Spectral Types for the HD stars (Ann Arbor, MI: Dept. of Astronomy, University of Michigan), QB6.H77

Jaschek, C., \& Jaschek, M. 1990, The classification of stars (Cambridge University Press)

Kharchenko, N. V., Piskunov, A. E., Roeser, S., Schilbach, E., \& Scholz R.-D. 2005, A\&A, 438, 1163

Kohoutek, L. 1971, A\&A, 13, 493

Kohoutek, L., \& Pauls, R. 1994, Astron. Nachr., 315, 409

Landolt, A. U. 1992, AJ, 104, 340

Mathis, J. S. 1990, ARA\&A, 28, 37

Mathys, G. 1988, A\&AS, 76, 427

Mathys, G. 1989, A\&AS, 81, 237

Megier, A., Krelowski J., Patriarchi, P., \& Aiello, S. 1997, MNRAS, 292, 853

Moffat, A. F. J., \& Vogt, N. 1975, A\&AS, 20, 125

Munch, L. 1955, Boletin de los Observatorios Tonantzintla y Tacubaya, 2, 28

Neckel, T. 1978, A\&A, 69, 51

Neckel, Th., \& Chini, R. 1981, A\&AS, 45, 451

Paladini, R., Burigana, C., Davies, R. D., et al. 2003, A\&A, 397, 213

Papaj, J., Wegner, W., \& Krelowski, J. 1991, MNRAS, 252, 403

Parker, Q. A., Phillips, S., Pierce, M. J., et al. 2005, MNRAS, 362, 689

Patriarchi, P., Morbidelli L., Perimotto M., \& Barbaro G. 2001, A\&A, 372, 644

Rieke G. H., \& Lebofsky M. J. 1985, ApJ, 288, 618

Russeil, D. 2003, A\&A, 397, 133

Russeil, D., Adami, C., \& Georgelin, Y. M. 2007, A\&A, 470, 161

Stock, J. 1984, Rev. Mex. Astron. Astrofis., 9, 127

Stupar, M., Parker, Q. A., \& Filipović, M. D. 2008, MNRAS, 390, 1037

Schaerer, D., \& de Koter, A. 1997, A\&A, 322, 598

Vogt, N., \& Moffat, A. F. J. 1975, A\&A, 45, 405

Walborn N. R. 1982, AJ, 87, 1300

Walborn N. R., \& Fitzpatrick, E. L. 1990, PASP, 102, 379

Wegner W. 1994, MNRAS, 270, 229

Yamaguchi, R., Saito, H., Mizuno, N., et al. 1999, PASJ, 51, 791

\section{Pages 5 to 9 are available in the electronic edition of the journal at http://www . aanda.org}


M. C. Pinheiro et al.: Distance determination of stars in Galactic H II regions $(R N)$

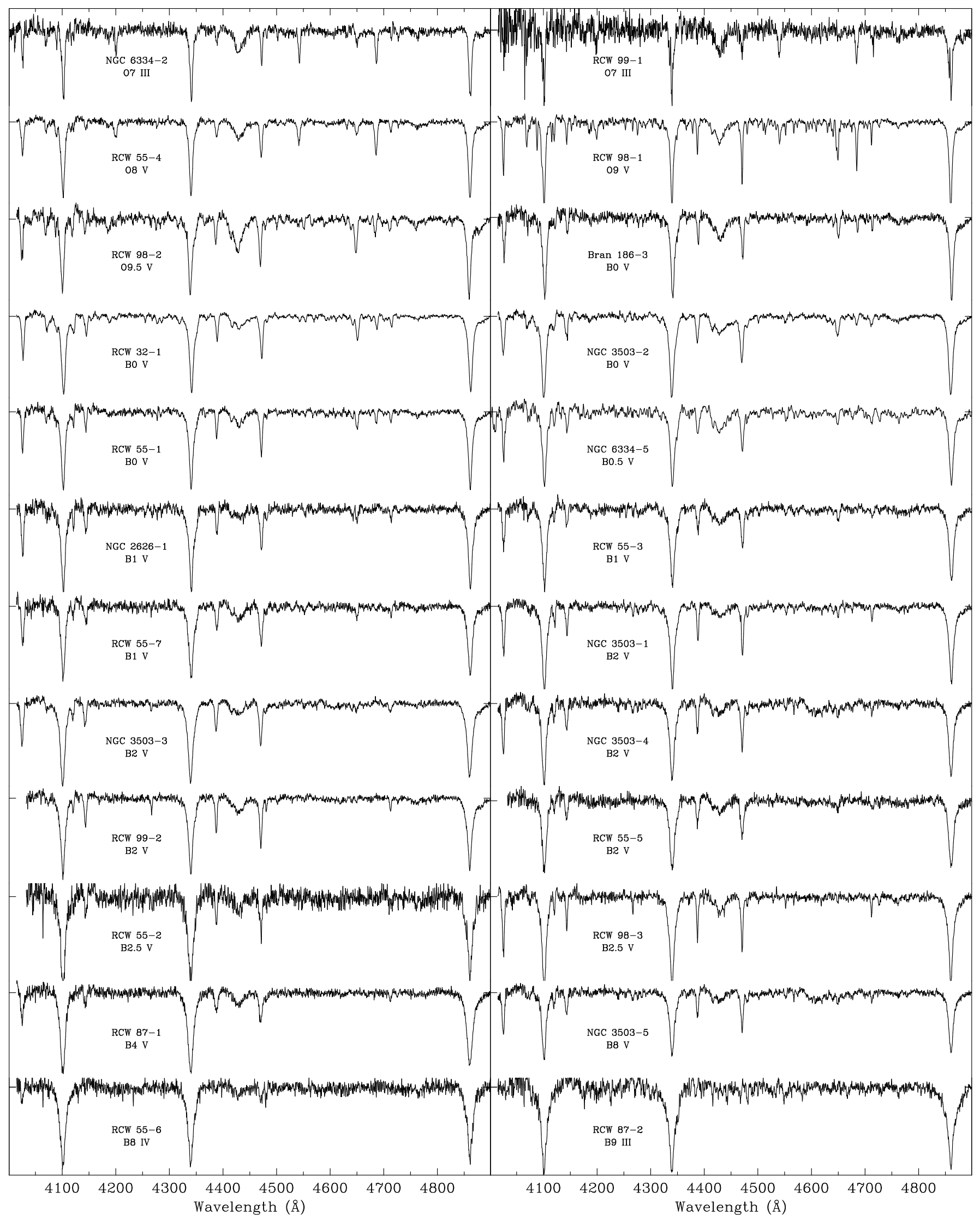

Fig. 1. Spectra of the OB stars. 


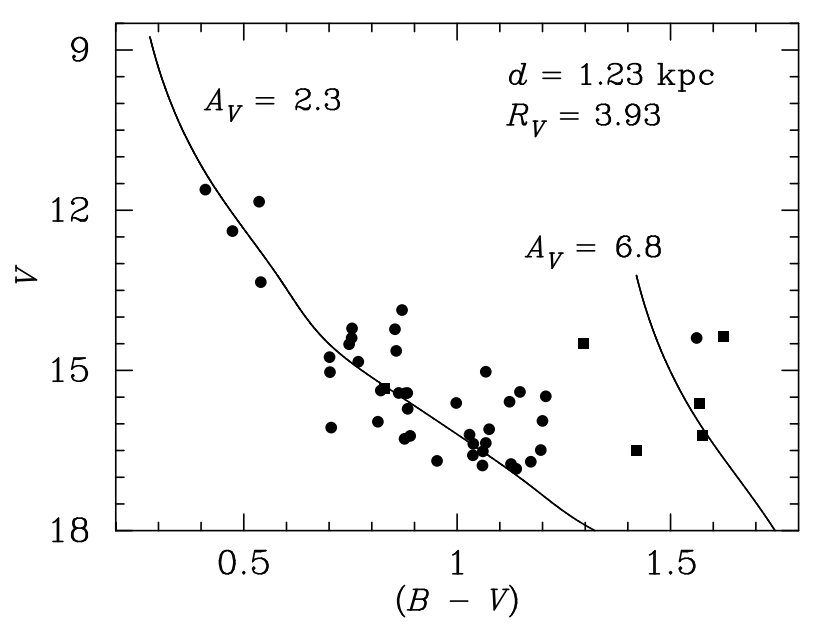

Fig. 2. The colour-magnitude diagram for RCW 87. The squares correspond to stars towards the embedded star cluster [BDB2003] G320.15+00.79 and the circles to the other stars. Also shown are the main sequence loci corresponding to a distance of $1.23 \mathrm{kpc}$ and two different reddening, $A_{V}=2.3$ and $A_{V}=6.8$.

Table 1. Journal for photometric observations.

\begin{tabular}{lllll}
\hline \hline H II region & \multicolumn{3}{c}{ Exposure (s) } & Date \\
\cline { 2 - 4 } & $U$ & $B$ & $V$ & \\
\hline RCW 55 & $3 \times 500$ & $4 \times 90$ & $3 \times 60$ & 2007 May 26 \\
NGC 3503 & & $5 \times 450$ & $5 \times 240$ & 2005 Apr. 13 \\
& & $5 \times 120$ & $5 \times 60$ & 2009 Mar 16 \\
RCW 87 & & $5 \times 450$ & $5 \times 240$ & 2005 Apr. 14 \\
& $9 \times 500$ & $5 \times 500$ & $3 \times 300$ & 2007 May 24 \\
RCW 98 & $6 \times 500$ & $9 \times 120$ & $10 \times 60$ & 2007 May 25 \\
& $6 \times 500$ & $5 \times 100$ & $7 \times 60$ & 2007 May 26 \\
RCW 99 & $6 \times 500$ & $5 \times 120$ & $5 \times 60$ & 2007 May 25 \\
Gum 61 & $9 \times 500$ & $5 \times 200$ & $5 \times 100$ & 2007 May 24 \\
Gum 64a & $4 \times 500$ & $5 \times 120$ & $5 \times 60$ & 2007 May 25 \\
\hline
\end{tabular}

Notes. $\dagger$ Part of NGC 6334. 
Table 2. Journal of spectroscopic observations.

\begin{tabular}{|c|c|c|c|c|c|c|c|c|c|c|}
\hline Star & $\alpha(2000)$ & $\delta(2000)$ & Tel. & Date & $\begin{array}{l}\text { Wavelength } \\
\text { range }(\AA)\end{array}$ & $\begin{array}{l}\text { Grid } \\
\text { gr./mm }\end{array}$ & $\begin{array}{l}\text { Scale } \\
((" / \mathrm{pxl})\end{array}$ & $\begin{array}{l}\text { Disp. } \\
(\AA ̊ \mathrm{~A} / \mathrm{pxl})\end{array}$ & $\begin{array}{l}\text { Res. } \\
(\AA)\end{array}$ & $\begin{array}{l}\text { Exp. } \\
\text { (s) }\end{array}$ \\
\hline \multirow[t]{2}{*}{ Bran 186-1 } & $08^{\mathrm{h}} 48^{\mathrm{m}} 43^{\mathrm{s}} .14$ & $-42^{\circ} 54^{\prime} 18^{\prime \prime} .3$ & $1.6 \mathrm{~m}$ & 2009 Mar 24 & $4015-4965$ & 1200 & 0.56 & 0.50 & 5.0 & $2 \times 600$ \\
\hline & & & $1.6 \mathrm{~m}$ & 2009 Mar. 24 & $4015-4965$ & 1200 & 0.56 & 0.50 & 2.7 & $1 \times 1200$ \\
\hline \multirow[t]{2}{*}{ Bran 186-2 } & 084848.14 & -425420.9 & $1.6 \mathrm{~m}$ & 2009 Mar. 24 & $4015-4965$ & 1200 & 0.56 & 0.50 & 2.7 & $2 \times 600$ \\
\hline & & & $1.6 \mathrm{~m}$ & 2009 Mar. 24 & $4015-4965$ & 1200 & 0.56 & 0.50 & 2.7 & $1 \times 1200$ \\
\hline \multirow[t]{2}{*}{ Bran 186-3 } & 084839.05 & -425354.0 & $1.6 \mathrm{~m}$ & 2009 Mar. 24 & $4015-4965$ & 1200 & 0.56 & 0.50 & 2.7 & $1 \times 1200$ \\
\hline & & & $1.6 \mathrm{~m}$ & 2009 Mar. 29 & $4015-4965$ & 1200 & 0.56 & 0.50 & 2.7 & $2 \times 1200$ \\
\hline Bran 186-4 & 084835.47 & -425431.5 & $1.6 \mathrm{~m}$ & 2009 Mar. 26 & $4015-4965$ & 1200 & 0.56 & 0.50 & 2.7 & $2 \times 1200$ \\
\hline Bran 186-5 & 084843.31 & -425433.1 & $1.6 \mathrm{~m}$ & 2009 Mar. 26 & $4015-4965$ & 1200 & 0.56 & 0.50 & 2.7 & $2 \times 1200$ \\
\hline NGC 2626-1 & 083531.44 & -404019.1 & $1.6 \mathrm{~m}$ & 2009 Mar. 27 & $4015-4965$ & 1200 & 0.56 & 0.50 & 2.7 & $1 \times 1200$ \\
\hline RCW 32-1 & 084440.33 & -411637.9 & $1.6 \mathrm{~m}$ & 2009 Mar. 27 & $4015-4965$ & 1200 & 0.56 & 0.50 & 2.7 & $3 \times 100$ \\
\hline \multirow[t]{3}{*}{ RCW 55-1 } & 105638.87 & -630102.8 & $1.6 \mathrm{~m}$ & 2009 Mar. 25 & $4015-4965$ & 1200 & 0.56 & 0.50 & 2.7 & $1 \times 1200$ \\
\hline & & & $1.6 \mathrm{~m}$ & 2009 Mar. 26 & $4015-4965$ & 1200 & 0.56 & 0.50 & 2.7 & $2 \times 1200$ \\
\hline & & & $1.6 \mathrm{~m}$ & 2009 Mar. 29 & $4015-4965$ & 1200 & 0.56 & 0.50 & 2.7 & $2 \times 1200$ \\
\hline \multirow[t]{3}{*}{ RCW 55-2 } & 105640.31 & -630104.5 & $1.6 \mathrm{~m}$ & 2009 Mar. 25 & $4015-4965$ & 1200 & 0.56 & 0.50 & 2.7 & $1 \times 1200$ \\
\hline & & & $1.6 \mathrm{~m}$ & 2009 Mar. 26 & $4015-4965$ & 1200 & 0.56 & 0.50 & 2.7 & $2 \times 1200$ \\
\hline & & & $1.6 \mathrm{~m}$ & 2009 Mar. 29 & $4015-4965$ & 1200 & 0.56 & 0.50 & 2.7 & $2 \times 1200$ \\
\hline \multirow[t]{2}{*}{ RCW 55-3 } & 105632.20 & -625958.5 & $1.6 \mathrm{~m}$ & 2008 Mar. 08 & $4000-6000$ & 600 & 0.51 & 1.05 & 5.0 & $1 \times 1200$ \\
\hline & & & $1.6 \mathrm{~m}$ & 2009 Mar. 26 & $4015-4965$ & 1200 & 0.56 & 0.50 & 2.7 & $3 \times 1200$ \\
\hline \multirow[t]{3}{*}{ RCW 55-4 } & 105642.20 & -630116.7 & $1.6 \mathrm{~m}$ & 2008 Mar. 08 & $4000-6000$ & 600 & 0.51 & 1.05 & 5.0 & $1 \times 600$ \\
\hline & & & $1.6 \mathrm{~m}$ & 2009 Mar. 25 & $4015-4965$ & 1200 & 0.56 & 0.50 & 2.7 & $1 \times 1200$ \\
\hline & & & $1.6 \mathrm{~m}$ & 2009 Mar. 29 & $4015-4965$ & 1200 & 0.56 & 0.50 & 2.7 & $1 \times 1200$ \\
\hline \multirow[t]{2}{*}{ RCW 55-5 } & 105640.16 & -625846.3 & $1.6 \mathrm{~m}$ & 2009 Mar. 25 & $4015-4965$ & 1200 & 0.56 & 0.50 & 2.7 & $1 \times 1200$ \\
\hline & & & $1.6 \mathrm{~m}$ & 2009 Mar. 25 & $4015-4965$ & 1200 & 0.56 & 0.50 & 2.7 & $2 \times 1200$ \\
\hline \multirow{3}{*}{ RCW 55-6 } & 105627.00 & -630000.4 & $1.6 \mathrm{~m}$ & 2008 Mar. 08 & $4000-6000$ & 600 & 0.51 & 1.05 & 5.0 & $1 \times 1200$ \\
\hline & & & $1.6 \mathrm{~m}$ & 2009 Mar. 26 & $4015-4965$ & 1200 & 0.56 & 0.50 & 2.7 & $3 \times 1200$ \\
\hline & & & $1.6 \mathrm{~m}$ & 2009 Mar. 29 & $4015-4965$ & 1200 & 0.56 & 0.50 & 2.7 & $1 \times 1200$ \\
\hline \multirow[t]{3}{*}{ RCW 55-7 } & 105632.28 & -630047.5 & $1.6 \mathrm{~m}$ & 2008 Mar. 08 & $4000-6000$ & 600 & 0.51 & 1.05 & 5.0 & $1 \times 1000$ \\
\hline & & & $1.6 \mathrm{~m}$ & 2009 Mar. 26 & $4015-4965$ & 1200 & 0.56 & 0.50 & 2.7 & $3 \times 1200$ \\
\hline & & & $1.6 \mathrm{~m}$ & 2009 Mar. 29 & $4015-4965$ & 1200 & 0.56 & 0.50 & 2.7 & $1 \times 1200$ \\
\hline \multirow[t]{2}{*}{ NGC 3503-1 } & 110117.17 & -595101.9 & $1.6 \mathrm{~m}$ & 2009 Mar. 24 & $4015-4965$ & 1200 & 0.56 & 0.50 & 2.7 & $1 \times 1200$ \\
\hline & & & $1.6 \mathrm{~m}$ & 2009 Mar. 29 & $4015-4965$ & 1200 & 0.56 & 0.50 & 2.7 & $1 \times 1200$ \\
\hline \multirow[t]{2}{*}{ NGC 3503-2 } & 110117.80 & -595030.3 & $1.6 \mathrm{~m}$ & 2008 Feb. 01 & $4000-5000$ & 1200 & 0.51 & 0.50 & 2.7 & $1 \times 1500$ \\
\hline & & & $1.6 \mathrm{~m}$ & 2009 Mar. 26 & $4015-4965$ & 1200 & 0.56 & 0.50 & 2.7 & $2 \times 1200$ \\
\hline \multirow[t]{2}{*}{ NGC 3503-3 } & 110118.24 & -595057.8 & $1.6 \mathrm{~m}$ & 2009 Mar. 24 & $4015-4965$ & 1200 & 0.56 & 0.50 & 2.7 & $1 \times 1200$ \\
\hline & & & $1.6 \mathrm{~m}$ & 2009 Mar. 29 & $4015-4965$ & 1200 & 0.56 & 0.50 & 2.7 & $1 \times 1200$ \\
\hline \multirow[t]{2}{*}{ NGC 3503-4 } & 110119.25 & -595056.6 & $1.6 \mathrm{~m}$ & 2009 Mar. 24 & $4015-4965$ & 1200 & 0.56 & 0.50 & 2.7 & $1 \times 1200$ \\
\hline & & & $1.6 \mathrm{~m}$ & 2009 Mar. 29 & $4015-4965$ & 1200 & 0.56 & 0.50 & 2.7 & $1 \times 1200$ \\
\hline NGC 3503-5 & 110059.27 & -595030.9 & $1.6 \mathrm{~m}$ & 2009 Mar. 26 & $4015-4965$ & 1200 & 0.56 & 0.50 & 2.7 & $2 \times 1200$ \\
\hline \multirow[t]{3}{*}{ RCW 87-1 } & 150509.78 & -573218.6 & $1.6 \mathrm{~m}$ & 2006 Apr. $24^{\dagger}$ & $3900-7800$ & 300 & 0.51 & 2.26 & 8.8 & $1 \times 1200$ \\
\hline & & & $1.6 \mathrm{~m}$ & 2009 Mar. 26 & $4015-4965$ & 1200 & 0.56 & 0.50 & 2.7 & $1 \times 1200$ \\
\hline & & & $1.6 \mathrm{~m}$ & 2009 Mar. 29 & $4015-4965$ & 1200 & 0.56 & 0.50 & 2.7 & $1 \times 1200$ \\
\hline RCW 87-2 & 150536.05 & -573258.8 & $1.6 \mathrm{~m}$ & 2009 Mar. 29 & $4015-4965$ & 1200 & 0.56 & 0.50 & 2.7 & $1 \times 1200$ \\
\hline RCW 87-3 & 150515.28 & -573125.1 & $1.6 \mathrm{~m}$ & 2009 Mar. 29 & $4015-4965$ & 1200 & 0.56 & 0.50 & 2.7 & $1 \times 1200$ \\
\hline \multirow[t]{2}{*}{ RCW 98-1 } & 155539.56 & -543836.5 & $1.6 \mathrm{~m}$ & 2008 Mar. 07 & $4000-6000$ & 600 & 0.51 & 1.05 & 5.0 & $1 \times 1200$ \\
\hline & & & $1.6 \mathrm{~m}$ & 2009 Mar. 26 & $4015-4965$ & 1200 & 0.56 & 0.50 & 2.7 & $3 \times 1000$ \\
\hline RCW 98-2 & 155540.97 & -544113.9 & $1.6 \mathrm{~m}$ & 2009 Mar. 26 & $4015-4965$ & 1200 & 0.56 & 0.50 & 2.7 & $3 \times 2000$ \\
\hline RCW 98-3 & 155542.64 & -543901.8 & $1.6 \mathrm{~m}$ & 2009 Mar. 26 & $4015-4965$ & 1200 & 0.56 & 0.50 & 2.7 & $3 \times 2000$ \\
\hline RCW 99-1 & 155938.44 & -534515.0 & $1.6 \mathrm{~m}$ & 2009 Mar. 25 & $4015-4965$ & 1200 & 0.56 & 0.50 & 2.7 & $1 \times 1200$ \\
\hline & & & $1.6 \mathrm{~m}$ & 2009 Mar. 25 & $4015-4965$ & 1200 & 0.56 & 0.50 & 2.7 & $2 \times 2400$ \\
\hline RCW 99-2 & 155938.74 & -534444.1 & $1.6 \mathrm{~m}$ & 2009 Mar. 25 & $4015-4965$ & 1200 & 0.56 & 0.50 & 2.7 & $2 \times 1800$ \\
\hline RCW 99-3 & 155954.52 & -534446.8 & $1.6 \mathrm{~m}$ & 2009 Mar. 25 & $4015-4965$ & 1200 & 0.56 & 0.50 & 2.7 & $2 \times 1800$ \\
\hline RCW 99-4 & 155931.16 & -534432.5 & $1.6 \mathrm{~m}$ & 2009 Mar. 25 & $4015-4965$ & 1200 & 0.56 & 0.50 & 2.7 & $1 \times 900$ \\
\hline NGC 6334-2 & 171946.16 & -360552.2 & $0.6 \mathrm{~m}$ & 2008 Aug. 01 & $3950-4950$ & 1200 & 0.56 & 0.50 & 2.7 & $2 \times 2000$ \\
\hline NGC 6334-5 & 172005.09 & -355641.7 & $0.6 \mathrm{~m}$ & 2008 Aug. 04 & $3950-4950$ & 1200 & 0.56 & 0.50 & 2.7 & $2 \times 2000$ \\
\hline
\end{tabular}

Notes. ${ }^{\dagger}$ Archive observation. 


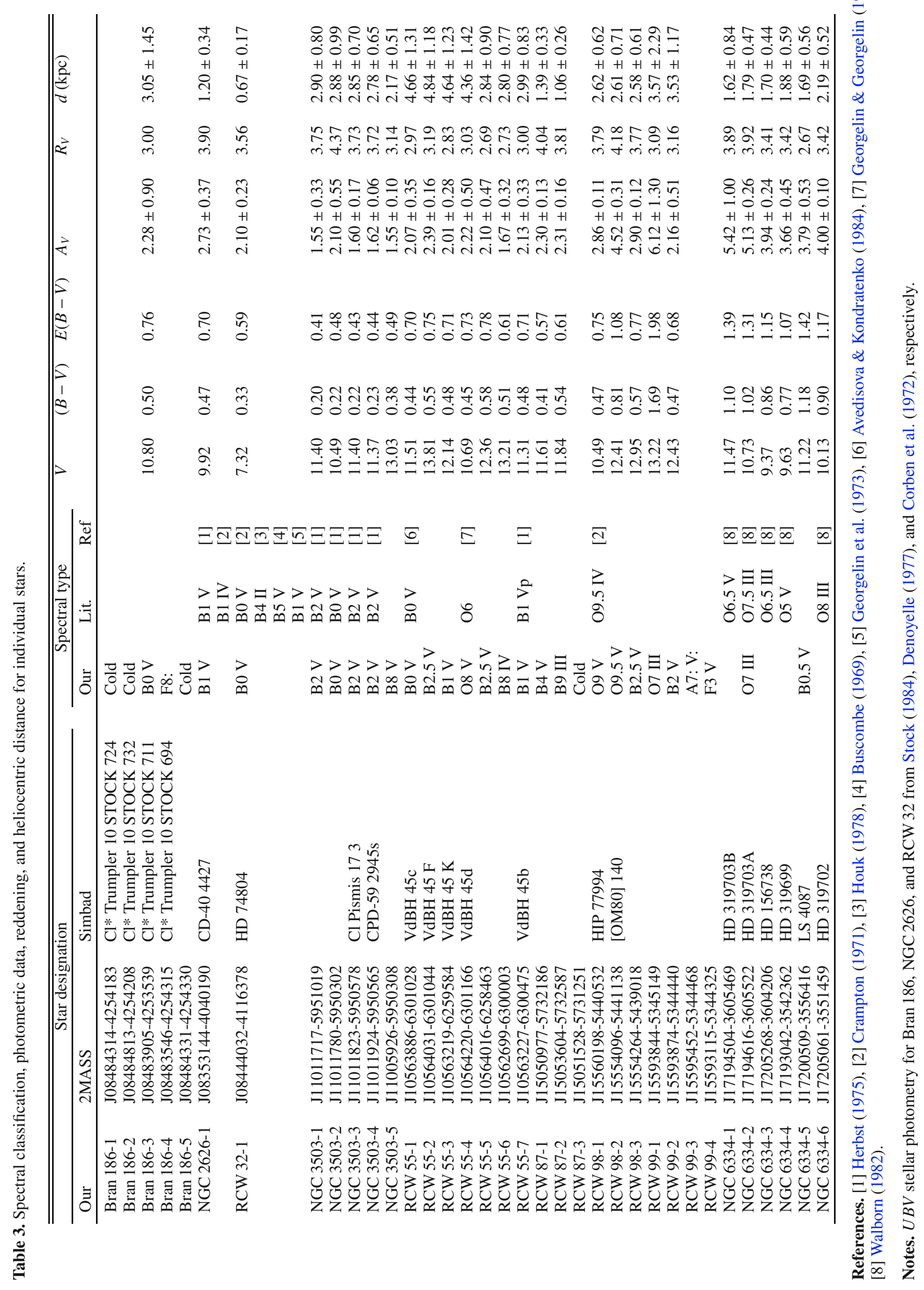

Page 8 of 9 
M. C. Pinheiro et al.: Distance determination of stars in Galactic H II regions $(R N)$

Table 5. Comparison between stellar and nebular properties.

\begin{tabular}{|c|c|c|c|c|c|c|c|c|c|}
\hline \multirow[t]{2}{*}{ H II region } & \multirow[t]{2}{*}{$d(\mathrm{kpc})$} & \multicolumn{3}{|c|}{$C(\mathrm{H} \beta)$} & \multicolumn{3}{|c|}{$\log N_{\mathrm{c}}\left(\right.$ photons s $\left.^{-1}\right)$} & \multirow[t]{2}{*}{$F(\lambda 4959) / \mathrm{H} \beta$} & \multirow[t]{2}{*}{ Spectral types } \\
\hline & & Stars & $\mathrm{H} \gamma / \mathrm{H} \beta$ & $2.7 \mathrm{GHz}$ & Stars & $\mathrm{H} \beta$ & $2.7 \mathrm{GHz}$ & & \\
\hline Bran 186 & 3.05 & 1.14 & 1.57 & & 48.02 & 47.2: & & 0.16 & B0 V \\
\hline NGC 3503 & 2.85 & 0.67 & 0.58 & 2.95 & 48.03 & 46.56 & 48.83 & 0.04 & B $0 \mathrm{~V}, 3 \times \mathrm{B} 2 \mathrm{~V}$ \\
\hline RCW 55 & 4.36 & 1.07 & 0.74 & & 48.90 & 46.82 & & 0.17 & $\mathrm{~B} 1 \mathrm{~V}$ \\
\hline RCW 87 & 1.23 & 2.70 & 2.89 & 3.34 & 45.16 & 47.44 & 48.08 & 0.31 & $\mathrm{~B} 4 \mathrm{~V}$ \\
\hline RCW 99 & 3.55 & 2.97 & 1.56 & 2.70 & 49.36 & 49.25 & 48.98 & 0.28 & O7 III, B2.5V \\
\hline Gum 64a & 1.75 & 2.13 & & & 47.77 & 47.81 & & & B $0.5 \mathrm{~V}$ \\
\hline
\end{tabular}

